\title{
Herpes simplex and herpes zoster viruses in COVID-19 patients
}

\author{
Joseph Katz ${ }^{1}$ (D) Sijia Yue ${ }^{2} \cdot$ Wei Xue $^{2}$
}

Received: 4 June 2021 / Accepted: 7 July 2021 / Published online: 11 July 2021

(c) Royal Academy of Medicine in Ireland 2021

\begin{abstract}
Background Reactivation of herpes family viruses in immunocompromised patients may result in detrimental outcomes for the hosts; therefore, herpes simplex virus-1 and varicella zoster virus infections in the context of COVID-19 may have clinical and prognostic implications.

Several reports associated this human herpes virus with COVID-19 infection and have claimed that it can be an indicator for latent COVID-19 infection. However, since most of these were case reports, it is impossible to assess the prevalence of these associations.

Methods The University of Florida patient registry $i 2 b 2$ with ICD-10 diagnosis codes was used for retrieval of patients with diagnosis of COVID-19 and each of the other viruses over the period of October 2015-June 2020.

Results The prevalence of the herpes simplex- 1 occurrence in the COVID- 19 group was $2.81 \%$ compared to $0.77 \%$ in the hospital population odds ratio of 5.27. When adjusted for gender, race, and age, the odds were 5.18, 4.48, and 4.61, respectively. After adjustment for respiratory disease, endocrine disease, obesity, diabetes, circulatory disease, and smoking, the odds were 1.94, 3.18, 1.37, 3.54, 3.7, and 5.1, respectively. The prevalence of the varicella zoster virus in COVID-19 patients was $1.8 \%$ compared to $0.43 \%$ in the hospital population, odds ratio of 5.26 before adjustment, and 5.2, 5.47, and 4.76 after adjusting for gender, age, and race, respectively. When adjusted for respiratory disease, endocrine disease, obesity, diabetes, and circulatory and neurological diseases, the odds were 1.3, 2.2, 1.48, 2.33, 2.85, and 2.6, respectively.
\end{abstract}

Conclusion Herpes simplex-1 and varicella zoster viruses are strongly associated with COVID-19 infection.

Keywords COVID-19 $\cdot$ Herpes simplex virus $\cdot$ Herpes zoster virus

\section{Introduction}

Herpes simplex 1 (HSV-1) and varicella zoster virus (VZV) are DNA viruses of the neurotropic alpha human herpesvirus subfamily (HHV) [1]. After the primary infection, these viruses can become dormant infections that can be reactivated when the immune system is compromised, resulting in significant damage to organs such as liver, kidney, and brain $[2,3]$.

Joseph Katz

jkatz@dental.ufl.edu

1 Department of Oral and Maxillofacial Diagnostic Sciences, University of Florida College of Dentistry, Gainesville, FL, USA

2 Department of Biostatistics, College of Public Health and Health Professionals, University of Florida, Gainesville, FL, USA
While few case reports have been published about the appearance of VZV and HSV in COVID-19 patients, the prevalence has not been established. Some studies have reported on cutaneous manifestations of COVID-19 such as eruptions, petechiae, and urticaria that are nonspecific and might have been related to the HHV viruses rather than to the SARS-COV-2 strain [3, 4].

Reactivation of the HHV due to the immunosuppressive state associated with COVID-19 could be potentially lifethreatening [5]. Therefore, it is important to investigate the prevalence of these occurrences and consider them in a differential diagnosis of HHV even when COVID-19 is confirmed. While there is no definitive treatment for COVID-19, there is a range of various anti-viral medications that are effective for HHV infections. Indeed, a few studies indicated that HHV are in fact responsible for the cutaneous manifestations and one study suggests that VZV might even be an indicator for latent COVID-19 infection [6]. 
In order to assess the prevalence of HSV and VZV in COVID-19 patients, we used the $i 2 b 2$ platform of the University of Florida patient registry to conduct a search of diagnoses for COVID-19, HSV, and VZV. Although retrospective cross-sectional studies cannot confirm causality between the 2 HHV and COVID-19, they can provide information about the prevalence and the strength of the association [7]. As far as we know, this is the first report on the prevalence of HSV and VZV in COVID-19-confirmed patients.

\section{Material and methods}

The study was exempted by the University of Florida (UF) institutional review board (IRB). The Integrated Data Repository (IDR) $i 2 b 2$ platform was used for the study. The study population included outpatients and inpatients attending the different UF Health centers across the state of Florida in the period of October 2015-June 2020. The database was searched for diagnostic codes ICD 10, COVID-19, ICD10U07.1, HSV-ICD10 B00, and VZV-ICD 10 B02. The codes represent a patient with a clinical diagnosis of COVID19, HSV-1 infection, and varicella zoster infection. The diagnosis of COVID-19 was confirmed by a PCR, and the HHV infection was established by antibody titers.

The IDR provided the total population, their age, and their sex distribution. The prevalence ratios were used to compare rates between groups. The odds ratios (ORs) for the associations were calculated by logistic regression of the aggregates using SAS statistical software. The $95 \%$ confidence interval (CI) and $P$-value for each OR were tabulated. $P<0.05$ was deemed significant. Since the platform enables to run only 3 variables at a time, we could not perform a multivariant regression and have adjusted each comorbidity at a time.

\section{Results}

A total of 889 patients with a confirmed diagnosis of COVID-19 were identified; 25 patients with both COVID19 and HSV-1 and 16 with COVID-19 and VZV were identified.

The prevalence of HSV-1 in the COVID-19 group was $2.81 \%$ compared to $0.77 \%$ in the hospital population (Table 1). This corresponded to OR of 5.23 before adjustments, 5.18 after gender adjustment, and 4.48 and 4.62 after

Table 1 Demographic information of patients with COVID 19, HSV, and HZV and hospital population (control)

\begin{tabular}{|c|c|c|c|c|c|c|}
\hline & HSV and COVID 19 & HSV in Hospital & HZV and COVID 19 & $\mathrm{HZV}$ in Hospital & COVID 19 & Hospital \\
\hline Total population & $25(2.81)$ & $7625(0.77)$ & $16(1.80)$ & $4228(0.43)$ & 889 & 987,849 \\
\hline$P$ value & $<0.00001 *$ & & $<0.00001 * *$ & & - & - \\
\hline \multicolumn{7}{|l|}{ Gender } \\
\hline Female & $19(76.00)$ & $5434(71.27)$ & $10(62.50)$ & $2632(62.25)$ & $509(57.26)$ & $532,391(53.89)$ \\
\hline Male & $6(24.00)$ & $2191(28.73)$ & $6(37.50)$ & $1596(37.75)$ & $380(43.42)$ & $455,458(46.11)$ \\
\hline Female/male & 3.16 & 2.48 & 1.66 & 1.64 & 1.32 & 1.16 \\
\hline \multicolumn{7}{|l|}{ Race } \\
\hline African American & $9(36.00)$ & $1525(20.00)$ & $6(37.50)$ & $601(14.21)$ & $228(25.60)$ & $111,627(11.30)$ \\
\hline Asian & $1(4.00)$ & $168(2.20)$ & $2(12.50)$ & $132(3.12)$ & $25(2.80)$ & $18,769(1.90)$ \\
\hline White & $15(60.00)$ & $5109(67.00)$ & $8(50.00)$ & $3189(75.43)$ & $400(45.00)$ & $493,923(50.00)$ \\
\hline Other & 0 & $823(10.7$ & 0 & $306(7.2)$ & $236(26.54)$ & $363,530(36.8)$ \\
\hline \multicolumn{7}{|l|}{ Age } \\
\hline $0-9$ & $0(0.00)$ & $389(5.10)$ & $0(0.00)$ & $22(0.52)$ & $17(1.90)$ & $90,881(9.20)$ \\
\hline $10-17$ & $0(0.00)$ & $290(3.80)$ & $0(0.00)$ & $20(0.47)$ & $22(2.47)$ & $66,186(6.70)$ \\
\hline $18-34$ & $14(56.00)$ & $2440(32.00)$ & $0(0.00)$ & $442(10.45)$ & 355 (39.90) & $212,388(21.50)$ \\
\hline $35-44$ & $9(36.00)$ & $1678(15.00)$ & $0(0.00)$ & $389(9.20)$ & $99(11.10)$ & $94,834(9.60)$ \\
\hline $45-54$ & $2(8.00)$ & $915(12.00)$ & $4(25.00)$ & $529(12.51)$ & $11(1.20)$ & $106,688(10.80)$ \\
\hline $55-64$ & $0(0.00)$ & $1029(13.50)$ & $6(37.50)$ & 926 (21.90) & $100(11.20)$ & $154,104(15.60)$ \\
\hline $65-74$ & $0(0.00)$ & $884(11.60)$ & $6(37.50)$ & 995 (23.53) & 109 (12.30) & $144,226(14.60)$ \\
\hline $74-85$ & $0(0.00)$ & $0(0.00)$ & $0(0.00)$ & $649(15.35)$ & $57(6.50)$ & 83,967 (8.50) \\
\hline$>85$ & 0 & 0 & 0 & 256 & 119 & 34,575 \\
\hline
\end{tabular}

*A comparison between the prevalence of HSV-1 in the COVID-19 group compared to the hospital group. The chi-square statistic is 46.4803 . Significant at $P<.05$

**A comparison between the prevalence of VZV in the COVID-19 group compared to the hospital group. The chi-square statistic is 38.2508 . Significant at $P<.05$ 
adjustment for race and age, respectively. The OR was still significant after we have adjusted the following comorbidities: respiratory disease, endocrine diseases, obesity, diabetes, circulatory disease, and smoking OR 1.94, 3.18, 1.37, $3.54,3.77$, and 5.1, respectively (Table 2 ). The prevalence of VZV in COVID-19 patients was $1.8 \%$ while that in the hospital was $0.43 \%$ (Table 1), corresponding to OR of 5.26 before adjustment and 5.2, 5.46, and 4.76 after adjustment for gender, race, and age, respectively. When adjusted for respiratory disease, endocrine disease, obesity, diabetes, circulatory disease, and neurological diseases, the ORs were $1.34,2.23,1.49,2,33,2.85$, and 2.616 , respectively (Table 2).

African American race and older age groups as compared to children were also at a higher risk to be affected by HHV (Table 2).

\section{Discussion}

In the present study, the prevalence of both HSV-1 and VZV was significantly increased in the COVID-19 group even after adjustments for comorbidities such as for respiratory disease, endocrine disease, obesity, diabetes, circulatory disease, and neurological disease; all have been recognized as significant risk factors for COVID-19 [8]. The significance of this finding is unclear as cross-sectional studies cannot relate causality in an association study. However, it is possible to speculate that COVID-19 lowers the threshold for reactivation of these common viruses. Xu et al. [5] reported the first case of reactivation of HSV in patients affected with COVID-19, and they assumed that HSV reactivation was related to the immunosuppression associated with COVID-19 infection. The onset of viremia (VZV and HSV-1) might have triggered the second septic shock and cytokine storm [5]. Le Balc'h [6] suggested that HHV reactivations are frequent in patients with COVID-19 acute respiratory disease, with higher rates in critically ill patients, and that HAV detection in the lower respiratory tract is associated with poorer outcomes. They have concluded that SARS-CoV-2 infection could be a risk factor for HHV reactivation and therefore recommend a rapid identification of these co-infections as it may impact the prognosis of infected patients [6].

Involvement of the central nervous system in COVID19 infection has been mentioned; however, only 2 cases of encephalitis caused by this infection have been reported. HSV-1 was reported to be associated with encephalitis in COVID-19 [9, 10].

Varicella-like exanthem was described as a specific COVID-19-associated skin manifestation [7]; however, it is not clear whether those were unique features of COVID-19 or VZV [11].
It is recommended that the differential diagnosis of COVID-19 associated with vesicular eruption should include disseminated HSV or VZV infections as well. HSV or VZV pneumonitis in particular may be especially detrimental. Marzano et al. considered varicella-like papulovesicular exanthem as a rare but specific disease COVID19-associated skin manifestation. They included patients with a COVID-19-positive nasopharyngeal swab and no medications with varicella-like lesions [11]. A previous case report of COVID-19-related varicella-like vesicles was also published by Recalcati [12]. Lamas-Velasco et al. [13] describe 3 cases that presented as papulovesicular exanthem in COVID-19 patients that were a combination of various HHV. They recommend using HHV family microarray PCR testing on the blister fluid of patients with COVID-19 with this type of eruptions.

Other complications of HHV in COVID-19 include a fatal case of acute liver failure due to HSV-1 infection [14] and three patients who have developed a necrotic herpes zoster on the second branch of the trigeminal nerve [15].

It has been suggested that VZV might be an indicator for latent COVID-19 [16], and although COVID-19 is known to affect the immune system and may increase the risk of VZV, only a limited number of reports have been published on the association between VZV and COVID-19 [15-17] and.

In the present study, the increased OR for COVID-19 with both viruses was diminished when adjusted for respiratory disease and obesity. Because HSV and cytomegalovirus (CMV) are the two viruses causing nosocomial viral pneumonia that can evolve into acute respiratory disease, our calculated OR for HSV in COVID-19 may be attributed to the presence of respiratory disease. Similarly, obesity has been linked to HHV infections.

We acknowledge that this study suffers from several weaknesses related to the nature of a patients' registry study. We did not have access to the detailed individual patient information. For example, the patients with COVID-19 may be sicker in general compared to the control hospitalized population, making them in general more likely to get viral reactivation. While there was control for comorbidities, there does not seem to be control for severity of acute illness or use of immune-suppressive medications which are strong risk factors for development of herpes infection. Nevertheless, we believe that the data is sufficient to establish a strong association between herpes viruses and COVID-19.

Approximately $36 \%$ of patients with HZV or HSV with COVID-19 were of the African American race. Although HSV prevalence recorded among non-Hispanic Blacks is significantly higher compared to White non-Hispanic, these findings may represent socioeconomic variables that were not addressed in this study. 
Table 2 Odds ratio for occurrence of HHV with COVID-19 before and after adjustments for co morbidities

\begin{tabular}{|c|c|c|c|c|}
\hline \multirow[b]{2}{*}{ HSV-1 vs no HSV-1* } & \multirow{2}{*}{$\begin{array}{l}\text { Odds ratio } \\
5.273\end{array}$} & \multicolumn{2}{|c|}{ 95\% Wald confidence limits } & \multirow{2}{*}{$\begin{array}{l}P \text { value } \\
<.0001\end{array}$} \\
\hline & & 3.541 & 7.851 & \\
\hline HSV-1 vs no HSV ${ }^{1}$ & 5.185 & 3.48 & 7.725 & $<.0001$ \\
\hline Male vs female & 0.908 & 0.795 & 1.038 & 0.157 \\
\hline HSV-1 vs no HSV- $1^{2}$ & 4.487 & 3.007 & 6.695 & $<.0001$ \\
\hline Black vs other & 3.337 & 2.788 & 3.993 & $<.0001$ \\
\hline White vs other & 1.271 & 1.086 & 1.486 & $<.0001$ \\
\hline HSV-1 vs no HSV- $1^{3}$ & 4.612 & 3.095 & 6.871 & $<.0001$ \\
\hline Age $18-34$ vs $0-17$ & 5.85 & 4.217 & 8.116 & $<.0001$ \\
\hline Age $>34$ vs $0-17$ & 2.936 & 2.128 & 4.052 & 0.0405 \\
\hline HSV-1 vs No HSV- $1^{4}$ & 1.946 & 1.283 & 2.953 & 0.0017 \\
\hline Respiratory disease vs no respiratory disease & 8.635 & 7.511 & 9.927 & $<.0001$ \\
\hline HSV-1 vs no HSV- $1^{5}$ & 3.181 & 2.174 & 4.655 & $<.0001$ \\
\hline Endocrine disease vs no endocrine disease & 4.264 & 3.733 & 4.87 & $<.0001$ \\
\hline HSV-1 vs no HSV- $1^{6}$ & 1.379 & 0.758 & 2.508 & 0.2919 \\
\hline Obesity vs no obesity & 5.705 & 4.91 & 6.63 & $<.0001$ \\
\hline HSV-1 vs no HSV- $1^{7}$ & 3.542 & 2.269 & 5.53 & $<.0001$ \\
\hline Diabetes vs no diabetes & 3.485 & 2.887 & 4.206 & $<.0001$ \\
\hline HSV-1 vs no HSV ${ }^{8}$ & 3.77 & 2.542 & 5.59 & $<.0001$ \\
\hline Circulatory disease vs no circulatory disease & 3.01 & 2.624 & 3.453 & $<.0001$ \\
\hline HSV-1 vs no HSV- $1^{9}$ & 5.101 & 3.339 & 7.793 & $<.0001$ \\
\hline Smokers vs never smoked & 0.976 & 0.822 & 1.158 & 0.7772 \\
\hline VZV vs no $\mathrm{VZV}^{*}$ & 5.266 & 3.105 & 8.931 & $<.0001$ \\
\hline $\mathrm{VZV}$ vs no $\mathrm{VZV}^{1}$ & 5.2 & 3.064 & 8.825 & $<.0001$ \\
\hline Male vs female & 0.886 & 0.776 & 1.012 & 0.0756 \\
\hline $\mathrm{VZV}$ vs no $\mathrm{VZV}^{2}$ & 5.476 & 3.224 & 9.302 & $<.0001$ \\
\hline Age $18-34$ vs $0-17$ & 5.475 & 3.988 & 7.517 & $<.0001$ \\
\hline Age $>34$ vs $0-17$ & 2.635 & 1.929 & 3.6 & 0.2 \\
\hline $\mathrm{VZV}$ vs no $\mathrm{VZV}^{3}$ & 4.76 & 2.803 & 8.084 & $<.0001$ \\
\hline Black vs other & 3.679 & 3.071 & 4.406 & $<.0001$ \\
\hline White vs other & 1.34 & 1.142 & 1.572 & $<.0001$ \\
\hline $\mathrm{VZV}$ vs no $\mathrm{VZV}^{4}$ & 1.341 & 0.694 & 2.591 & 0.383 \\
\hline Respiratory disease vs no respiratory disease & 8.542 & 7.436 & 9.813 & $<.0001$ \\
\hline VZV vs VZV 5 & 2.233 & 1.286 & 3.876 & 0.0043 \\
\hline Endocrine disease vs no endocrine disease & 4.247 & 3.719 & 4.849 & $<.0001$ \\
\hline $\mathrm{VZV}$ vs no $\mathrm{VZV}^{6}$ & 1.484 & 0.703 & 3.13 & 0.3002 \\
\hline Obesity vs no obesity & 5.556 & 4.778 & 6.46 & $<.0001$ \\
\hline VZV vs no $\mathrm{VZV}^{7}$ & 2.336 & 1.205 & 4.528 & 0.012 \\
\hline Diabetes vs no diabetes & 3.42 & 2.827 & 4.138 & $<.0001$ \\
\hline $\mathrm{VZV}$ vs no $\mathrm{VZV}^{8}$ & 2.851 & 1.673 & 4.858 & 0.0001 \\
\hline Circulatory disease vs no circulatory disease & 3.091 & 2.696 & 3.544 & $<.0001$ \\
\hline VZV vs no $\mathrm{VZV}^{9}$ & 2.616 & 1.506 & 4.544 & 0.0006 \\
\hline Neurological disease vs no neurological disease & 3.43 & 2.996 & 3.928 & $<.0001$ \\
\hline
\end{tabular}

* Raw models that evaluate the association between COVID-19 status with herpes and herpes zoster without adjusting for any covariate

${ }^{1}$ Adjusts for gender

${ }^{2}$ Adjusts for race

${ }^{3}$ Adjusts for age

${ }^{4}$ Adjusts for respiratory disease

${ }^{5}$ Adjusts for endocrine disease

${ }^{6}$ Adjusts for obesity

${ }^{7}$ Adjusts for diabetes

${ }^{8}$ Adjusts for circulatory disease

${ }^{9}$ Adjust for smoking and neurological disease 
In conclusion, in the present study we have demonstrated a strong association between herpes virus infection and COVID-19. Although the exact nature of the association is yet to be elucidated, clinicians should be knowledgeable about it. Both herpes viruses and COVID-19 may affect many organs and systems. Keeping in mind that fulminant herpes infection may resemble COVID-19 in some cases, clinicians should include herpes in the differential diagnosis of these cases especially because of the broad availability of anti-herpetic medications, an option still nonexistent for COVID-19 infection.

Author contribution Joseph Katz-conceptualization, writing of manuscript, and data analysis; Sijia Yue — data analysis; Wei Xue—statistical analysis, data interpretation.

Availability of data and materials May be provided by request.

\section{Declarations}

Ethics approval The study was determined exempted by the University of Florida Institutional Review Board.

Consent to participate All authors have agreed to participate.

Consent for publication All authors have agreed in the publication.

Conflict of interest The authors declare no competing interests.

\section{References}

1. Ouwendijk WJ, Laing KJ, Verjans GM, Koelle DM (2013) T-cell immunity to human alphaherpesviruses. Curr Opin Virol $3: 452-460$

2. Mitchell BM, Bloom DC, Cohrs RJ et al (2003) Herpes simplex virus-1 and varicella-zoster virus latency in ganglia. J Neurovirol 9:194-204

3. Grant-Kels JM, Sloan B, Kantor J, Elston DM (2020) Big data and cutaneous manifestations of COVID-19. J Am Acad Dermatol 83(2):365-366. https://doi.org/10.1016/j.jaad.2020.04.050

4. Lewis DJ, Schlichte MJ, Dao H Jr (2017) Atypical disseminated herpes zoster: management guidelines in immunocompromised patients. Cutis 100(5):321-330
5. Xu R, Zhou Y, Cai L et al (2020) Co-reactivation of human herpesvirus alpha subfamily (HSV I and VZV) in critically ill patient with COVID-19. Br J Dermatol 183(6):1145-1147. https://doi. org/10.1111/bjd.19484

6. Le Balc'h P, Pinceaux K, Pronier C et al (2020) Herpes simplex virus and cytomegalovirus reactivations among severe COVID-19 patients. Crit Care 24(1):530. https://doi.org/10.1186/ s13054-020-03252-3

7. Levin KA (2006) Study design III: cross-sectional studies. Evid Based Dent 7(1):24-25. https://doi.org/10.1038/sj.ebd.6400375

8. Ye M, Ren Y, Lv T (2020) Encephalitis as a clinical manifestation of COVID-19. Brain Behav Immun 88:945-946. https://doi.org/ 10.1016/j.bbi.2020.04.017

9. Zheng Z, Peng F, Xu B et al (2020) Risk factors of critical \& mortal COVID-19 cases: a systematic literature review and metaanalysis. J Infect 81(2):e16-e25. https://doi.org/10.1016/j.jinf. 2020.04.021

10. Lovati C, Osio M, Pantoni L (2020) Diagnosing herpes simplex-1 encephalitis at the time of COVID-pandemic. Neurol Sci 41(6):1361-1364

11. Marzano AV, Genovese G, Fabbrocini G et al (2020) Varicella-like exanthem as a specific COVID- 19- associated skin manifestation: multicenter case series of 22 patients. J Am Acad Dermatol 83(1):280-285. https://doi.org/10.1016/j.jaad.2020.04.044

12. Recalcati S, Fantini F (2020) Chilblain-like lesions during the COVID-19 pandemic: early or late sign? Int J Dermatol 59(8):e268-e269. https://doi.org/10.1111/ijd.14975

13. lamas-Velasco M, Rodríguez-Jiménez P, Chicharro P, (2020) Reply to "Varicella-like exanthem as a specific COVID19-associated skin manifestation: multicenter case series of 22 patients": to consider varicella-like exanthem associated with COVID-19 virus varicella zoster and virus herpes simplex must be ruled out. J Am Acad Dermatol 83(3):e253-e254. https://doi. org/10.1016/j.jaad.2020.04.180

14. Busani S, Bedini A, Biagioni E et al (2020) Two fatal cases of acute liver failure due to HSV-1 infection in COVID-19 patients following immunomodulatory therapies. Clin Infect Dis 73(1):e252-e255. https://doi.org/10.1093/cid/ciaa1246

15. Tartari F, Spadotto A, Zengarini C et al (2020) Herpes zoster in COVID-19-positive patients. Int J Dermatol 59(8):1028-1029. https://doi.org/10.1111/ijd.15001

16. Elsaie ML, Nada HA (2020) Herpes Zoster (shingles) complicating the course of COVID19 infection. J Dermatolog Treat 1-7

17. Pona A, Jiwani RA, Afriyie F et al (2020) Herpes zoster as a potential complication of coronavirus disease 2019. Dermatol Ther 33(6):e13930. https://doi.org/10.1111/dth.13930 